\title{
Occupational exposure to organic solvents as a cause of sleep apnoea
}

\author{
Christer Edling, Arne Lindberg, Jan Ulfberg
}

\begin{abstract}
A high prevalence of sleep apnoea was found in a group of men occupationally exposed to organic solvents. Workers with long term exposure to organic solvents often report symptoms such as fatigue, forgetfulness, and concentration difficulties. These symptoms are strikingly similar to those reported by patients with obstructive sleep apnoea syndrome (OSAS). This is a frequently diagnosed disorder characterised by disturbed sleep causing psychic or somatic complications and daytime sleepiness. A study was undertaken to evaluate whether people with long term occupational exposure to organic solvents have a higher prevalence of sleep apnoea than the general population. Patients exposed to solvents $(66 \mathrm{men})$ were invited to participate in a screening for sleep apnoea. A static charge sensitive bed was used for the monitoring of respiration movements and pulse oximetry during one night. A classical sleep apnoea was diagnosed if periodic respiration movement exceeded $45 \%$ of estimated sleep time and the oxygen desaturation index exceeded 6 . The prevalence of sleep apnoea among the men exposed to solvents was compared with the prevalence in the general population $(1 \cdot 4 \%)$. The prevalence among the participating exposed men was $19.7 \%$ which gave a conservative relative risk estimate of $14 \cdot 1$ (95\% confidence interval $(95 \% \mathrm{CI}) \mathbf{7 \cdot 5 - 2 4 \cdot 2 )}$. The results indicate that exposure to organic solvents causes sleep apnoea. An alternative possibility is that people with sleep apnoea are misdiagnosed as cases of solvent induced toxic encephalopathy. The interpretation has importance for the caring of the patient.
\end{abstract}

(British fournal of Industrial Medicine 1993;50:276-279)

Department of Occupational Medicine, University Hospital, S-751 85 Uppsala, Sweden

C Edling

Department of Internal Medicine, Avesta Hospital, Avesta

A Lindberg, J Ulfberg
Occupational exposure to organic solvents might cause acute and chronic effects on the central nervous system. Chronic effects, for instance, toxic encephalopathy, have been recognised for many years as an occupational disease in the Scandinavian countries. ${ }^{1}$ During recent years chronic effects have also been reported from other countries. ${ }^{2-4}$ Workers with long term exposure to organic solvents often report symptoms such as fatigue, forgetfulness, and concentration difficulties. These symptoms are strikingly similar to those reported by patients with sleep apnoea. ${ }^{5}$ The obstructive sleep apnoea syndrome (OSAS) is a frequently diagnosed disorder that is characterised by loud snoring and repeated upper airway obstructions during sleep that cause psychic or somatic complications. Patients with OSAS usually have disturbed sleep and complain of daytime sleepiness. Most patients with OSAS are obese, and sometimes there is a specific cause of OSAS predisposing to a narrowing of upper airways. Several studies have suggested a comorbidity between habitual snoring, OSAS, and vascular diseases. A high occurrence of sleep apnoea is reported in various diseases of the central nervous system, such as senile dementia of the Alzheimer type, brain stèm disorders, as a sequel after encephalitis, and after head trauma, as well as diseases of the autonomic nervous system. There is also a slight increase in the number of sleep apnoeas with advancing age and among patients with hypothyroidism. In three studies exposure to organic solvents has been implicated as a possible cause of sleep apnoea. ${ }^{678}$

This study was undertaken to evaluate whether people with long term occupational exposure to organic solvents have a higher prevalence of sleep apnoea than the general population.

Material and methods

The study group comprised all patients from Kopparberg county occupationally exposed to solvents and referred to the Department of Occupational Medicine in Uppsala during 1984 to 1988 ; in total 66 men. We sent a questionnaire to 
their home addresses, regarding sleep problems and an invitation to participate in a sleep apnoea screening at the Department of Medicine, Avesta Hospital.

The medical history, body mass index (BMI), and blood pressure was recorded for each man. We used a static charge sensitive bed for the monitoring of respiration movements and pulse oximetry during one night. ${ }^{8}$ The respiration movements were recorded on paper, and the number of pathological recordings calculated. A pathological 'sleep recording was defined as more than five apnoeas a night. If periodic respiration movement exceeded $45 \%$ of estimated sleep time and the oxygen desaturation index exceeded 5 the recording was classified as a classical sleep apnoea. ${ }^{9}$

The prevalence of sleep apnoea among the men exposed to solvent was compared with the prevalence in the general population, obtained from an epidemiological study of the prevalence of sleep apnoea syndrome among Swedish men in the age group 30 to $69 .^{10}$

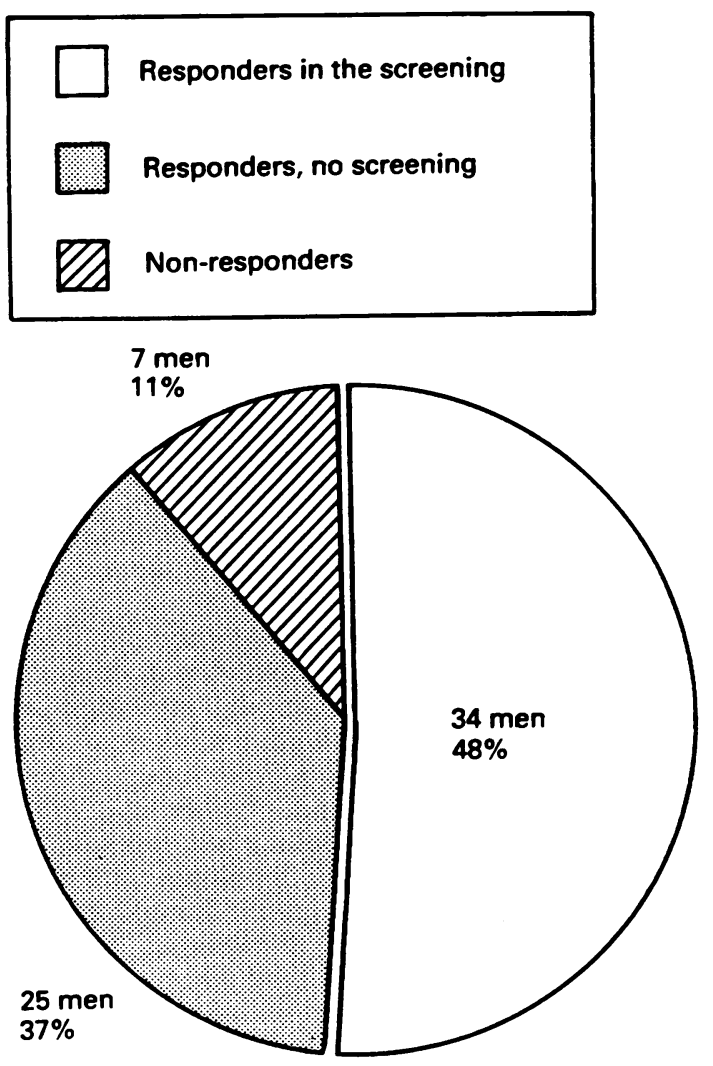

Figure 1 Responders and non-responders $(n=66)$ and number of participants in screening for sleep apnoea.

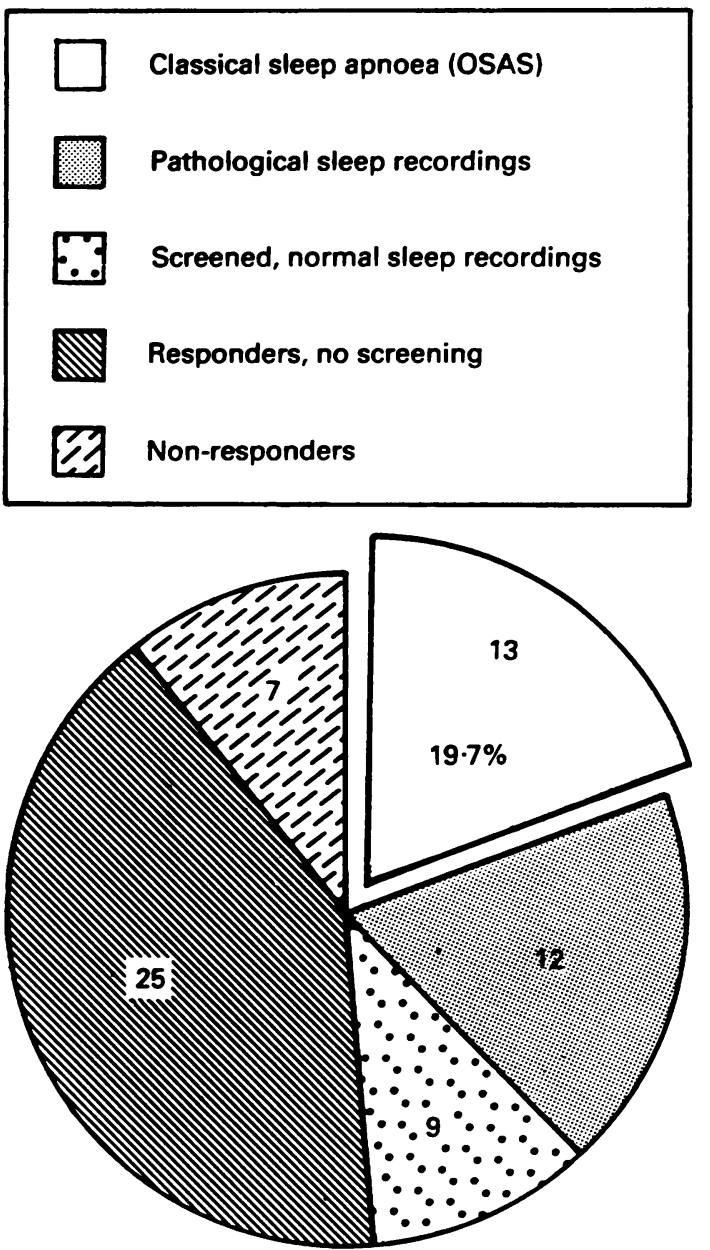

Figure 2 Number of men with OSAS, pathological sleep recordings, normal sleep recordings, and non-participants in screening for sleep apnoea.

\section{Results}

Of the 66 men, $59(89 \%)$ answered the questionnaire and $34(52 \%)$ took part in the screening study (fig 1). The mean age for the participants was 53 and mean exposure time to solvents was 24 years. Corresponding figures for the non-participants were 49 and 21 years respectively. Of the 34 men, 25 had pathological sleep recordings, and only nine $(14 \%)$ were normal. Of those with pathological recordings, 13 had a classical sleep apnoea (fig 2). This gave a prevalence of $19.7 \%$ among the entire study group of 66 men, assuming the non-participants had normal sleep recordings. The apnoeas were of the obstructive type. The BMI was $26 \cdot 0$ among those with normal sleep recordings, $27 \cdot 5$ 


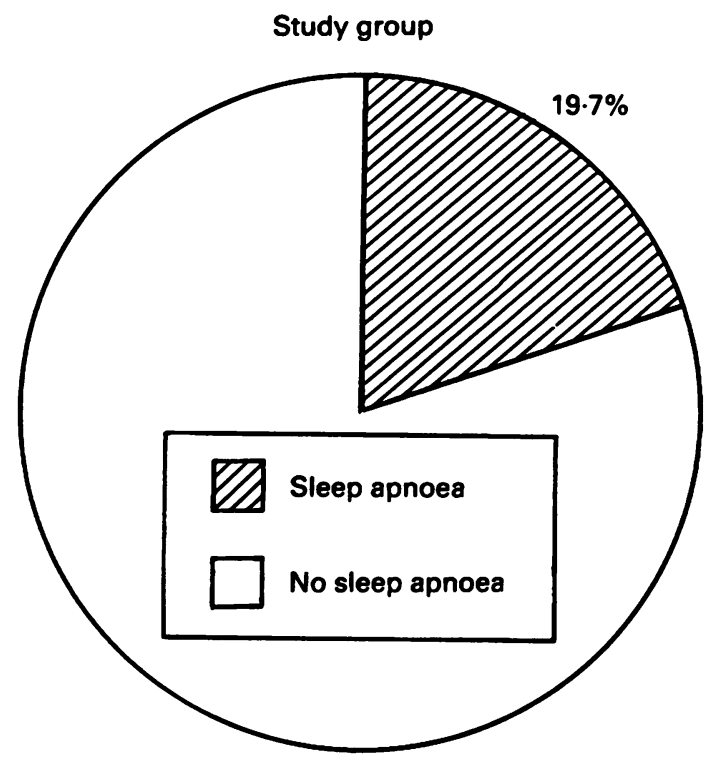

Swedish men aged $30-69$ years

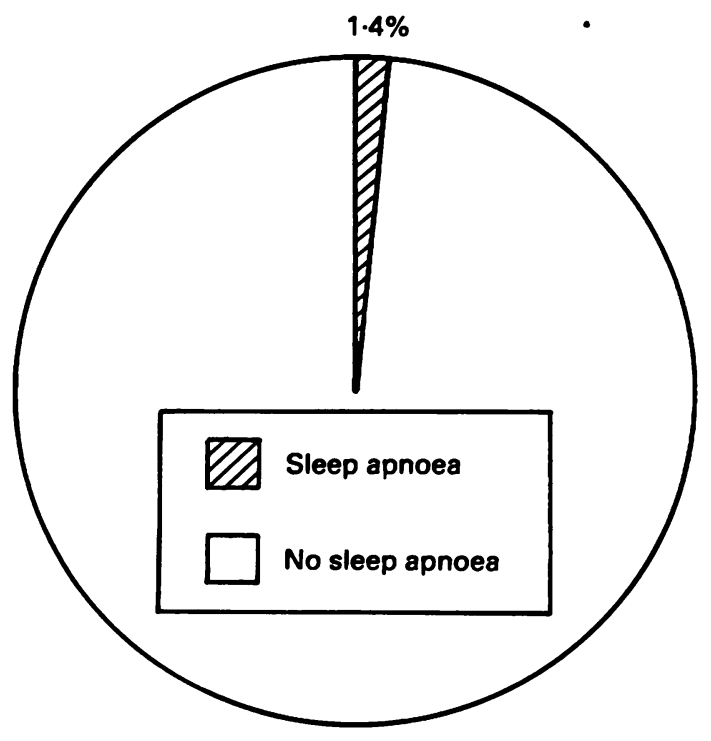

$R R=\frac{19 \cdot 7}{1 \cdot 4}=14 \cdot 1$

$(95 \% \mathrm{Cl} 7 \cdot 5-24 \cdot 2)$

Figure 3 Prevalence of sleep apnoea among Swedish men and the study group. among those with OSAS, and 25.5 among those with pathological sleep recordings but no OSAS. There was no difference in mean blood pressure between the three groups.

The prevalence in the general population of Swedish men of the same age group is $1.4 \%$. As the prevalence among the exposed men was $19 \cdot 7 \%$ the conservative relative risk estimate was $14 \cdot 1$ with a $95 \%$ confidence interval $(95 \% \mathrm{CI})$ of $7 \cdot 5-24 \cdot 2$ (fig 3).

Further analyses of exposure time showed a relative risk for sleep apnoea of $1.3(95 \%$ CI $0.3-4 \cdot 6)$ for those exposed for 20 years or more, compared with those with less than 20 years of exposure. No man with less than 10 years of exposure experienced sleep apnoea.

\section{Discussion}

A high prevalence of sleep apnoea was found in a group of men occupationally exposed to organic solvents. This might indicate that exposure to organic solvents causes sleep apnoea. An alternative possibility is that people with sleep apnoea are misdiagnosed as cases of solvent induced toxic encephalopathy.

The study group comprised patients referred to the Department of Occupational Medicine in Uppsala, due to occupational exposure to organic solvents. This might have entailed a selection bias and decreased the internal validity of the study. Although most of the men were referred on the basis of symptoms such as fatigue, forgetfulness, and concentration difficulties, none of the patients were referred due to a suspicion of a sleep apnopea syndrome. It might be argued that if the doctor had diagnosed a possible sleep apnoea syndrome, the patient would have been referred to another department. This might actually lead to an underestimation of the true prevalence among the exposed people in our study.

The single factor most commonly associated with OSAS is obesity. ${ }^{5}$ The BMI in our study did not, however, show any statistically significant difference between the men with pathological sleep recordings and those with normal sleep pattern. Although there was a somewhat higher BMI in the group with OSAS, the difference was too small to explain the increased prevalence. Neither was there any difference between the groups for blood pressure; nor were there clinical signs of hypothyroidism. We therefore believe that the most common and recognised causes of OSAS can be ruled out as explanations for our results.

In evaluating exposure to organic solvents as a cause of OSAS, assessment of exposure must be accurate. In this study we had access to histories of 
occupational exposure taken by experienced occupational physicians and hygienists. Nevertheless, it was not possible to classify exposure into categories other than exposure time and type of solvents, because specific exposure measurements were missing in most cases. There was a slight dose-response relation and men with more than 20 years of exposure had a higher prevalence of OSAS than men with shorter exposure times. The findings that no man with less than 10 years of exposure experienced sleep apnoea might imply a threshold of minimum exposure time, an interval identical to that suggested for toxic encephalopathy induced by organic solvents. ${ }^{1}$ Concerning type of solvent it was not possible to identify a certain solvent or work task, as most of the men were exposed to mixtures of organic solvents in their work as house painters, spray painters, degreasers, floor layers, petrol fillers etc. There was no difference in the prevalence of OSAS among exposed men regardless of toxic encephalopathic diagnosis.

Sleep apnoea among workers exposed to solvents has earlier been reported in two studies. In a case report from the United States ${ }^{6}$ a patient with exposure to trichloroethane had a severe sleep apnoea of the central type. In two Norwegian studies 78 workers exposed to trichloroethane and other organic solvents had a significantly increased number of sleep apnoeas compared with unexposed controls. The apnoeas were mainly of the obstructive type. Our results accord with the Norwegian study and support their conclusion that there is a strong interrelation between exposure to organic solvents and the sleep apnoea syndrome.

Sleep difficulties are common and can cause severe social problems. In Sweden about $15 \%$ of men report habitual snoring and $10 \%$ of these have OSAS. It is important to elucidate whether expo- sure to organic solvents is a cause of OSAS, because such a finding will have important implications for prevention. On the other hand, if OSAS is wrongly diagnosed as a toxic encephalopathy among workers occupationally exposed to organic solvents this could mean that these patients are incorrectly treated. In the first alternative the efforts would normally be directed toward reducing exposure, whereas the OSAS might be treated and cured by other means-for example, surgery.

1 Flodin U, Edling C, Axelson O. Clinical studies of psychoorganic syndrome among workers with exposure to solvents. Am f Ind Med 1984;5:297-95.

2 Brackbill RM, Maizlish N, Fisschbach T. Risk of neuropsychiatric disability among painters in the United States. Scand $\mathcal{f}$ Work Environ Health 1990;16:182-8.

3 Gubéran E, Usel M, Raymond L, Tissot R, Sweetnam PM. Disability, mortality, and incidence of cancer among Geneva painters and electricians: a historical prospective study. $\mathrm{Br} \boldsymbol{f}$ Ind Med 1989;46:16-23.

4 van Vliet C, Swaen GMH, Volovics A, Tweehuysen M, Meijers JMM, de Boorder T, Sturmans F. Neuropsychiatric disorders among solvent-exposed workers. First results from a Dutch case-control study. Int Arch Occup Environ Health 1990;62:127-32.

5 Guilleminault C, Partinen M, eds. Obstructive sleep apnoea syndrome: clinical research and treatment. New York; Raven Press 1990.

6 Wise MG, Fisher JG, de la Plata A. Trichloroethane (TCE) and central sleep apnoea: A case study. $\mathcal{F}$ Toxicol Environ Health 1983;11:101-4.

7 Monstad P, Nissen T, Sulg IA, Mellgren SJ. Sleep apnoea and organic solvent exposure. $\mathcal{F}$ Neurol 1987;234:152-4.

8 Monstad P, Mellgren SI, Sulg JA. The clinical significance of sleep apnoea in workers exposed to organic solvents: implications for the diagnosis of organic solvent encephalopathy. f Neurol 1992;239:195-8.

9 Svanborg E, Larsson H, Carlsson-Nordlander B, Pirskanen R. A limited diagnostic investigation for obstructive sleep apnoea syndrome. Oximetry and static charge sensitive bed. Chest 1990;98:1341-5.

10 Gislason T, Almqvist M, Eriksson G, Taube A, Boman G. Prevalence of sleep apnoea among Swedish men-an epidemiological study. 7 Clin Epidemiol 1988;41:571-6.

Accepted 8 June 1992. 